\title{
A INADEQUAÇÃO DOS EXAMES RADIOLÓGICOS PERIÓDICOS INDISCRIMINADOS EM SAUUE OCUPACIONAL: RESULTADOS DO CENSO DE 1984 EM UMA EMPRESA DE GRANDE PORTE
}

Eduardo Algranti*

Salim Amed Ali**

Aloisio Punhagui Cuginotti***

ALGRANTI, E. et al. A inadequação dos exames radiológicos periódicos indiscriminados em saúde ocupacional: resultados do censo de 1984 em uma empresa de grande porte. Rev. Saúde públ., S. Paulo, 20:26-32, 1986

RESUMO: São aqui relatados os resultados do censo abreugráfico de 1984 de uma empresa de grande porte, isenta de riscos ocupacionais para o aparelho respiratório. Em 5.308 abreugrafias realizadas foram detectados 53 casos de anormalidades abreugráficas $(1 \%)$, dos quais apenas 4 de tuberculose ativa. O rendimento foi de $0,75 / 1.000$ abreugrafias. $O$ custo médio de deteç̧ão de cada caso de tuberculose ativa foi de 256,32 ORTN (ao redor de 1.850 dólares). Foi também constatado um menor comparecimento de funcionários ao exame abreugráfico periódico, $1,6 \%$ de ausências em 1982 e 7,8\% em 1984, causado provavelmente pelo crescente conhecimento leigo sobre radiaçōes ionizantes. Os resultados são discutidos e é apresentada uma revisão sobre o emprego de exames radiológicos periódicos em saúde ocupacional.

UNITERMOS: Abreugrafia, Radiação ionizante. Saúde ocupacional.

\section{INTRODUÇÃO}

Os exames abreugráficos constantes em lei, no Brasil, vêm sendo questionados em relação à sua utilização indiscriminada na população economicamente ativa. Este questionamento decorre de experiência acumulada nas últimas décadas, desde a proposta inicial de Manoel de Abreu da "Carteira de Saúde Universal" ${ }^{1}$. Pela facilidade de execução, rapidez nos resultados e menor custo, a abreugrafia ganhou aceitação geral, tornando-se exame rotineiro, mesmo em crianças e mulheres grávidas. O conhecimento sobre as consequiências das radiações aprofundou-se após as explosões atômicas em Hiroshima e Nagasaki. Doses mínimas de radiação podem constituir sérios riscos somáticos e genéticos. Desse modo, a utilização sistemática de abreugrafia, como exame de massa, passou a constituir séria preocupação para os profissionais ligados a área radiológica, clínica e ocupacional.

A atual legislação trabalhista prevê exames médicos e abreugráficos (em localidades onde houver) admissionais, periódicos e demissionais, custeados pelo empregador ${ }^{14}$. A abreugrafia deverá ser substituída pela telerradiografia nas atividades especificadas na NR-15 ${ }^{13}$ sendo que a primeira deverá ser renovada a cada 2 anos e a segunda anualmente.

A Secretaria de Relações do Trabalho e a Secretaria de Segurança e Medicina do Trabalho, do Ministério do Trabalho, determinaram aos órgãos competentes de fiscalização que suspendessem a exigên-

\footnotetext{
- Da Fundaçăo Jorge Duprat Figueiredo de Segurança e Medicina do Trabalho (FUNDACENTRO) - Rua Capote Valente, 710 - 05409 - São Paulo, SP - Brasil e do Hospital dos Servidores Públicos do Estado de Sảo Paulo - Rua Pedro de Toledo, 1800-04039- Sáo Paulo, SP - Brasil

* Da Fundação Jorge Duprat Figueiredo de Segurança e Medicina do Trabalho (FUNDACENTRo) Rua Capote Valente, 710 - 05409 - São Paulo, SP - Brasil.

** Do Instituto Nacional de Assistencia Médica e Previdência Social - Hospital Ipiranga - Av. Nazaré, 28 - 04262 - Săo Paulo, SP - Brasil.
} 
ALGRANTI, E. et al. A inadequação dos exames radiológicos periódicos indiscriminados em saúde ocupacional: resultados do censo de $1984 \mathrm{em}$ uma empresa de grande porte. Rev. Saúde públ., S. Paulo, 20: 26-32, 1986.

cia de exames abreugráficos, tanto admissionais quanto periódicos*

O aparelho de abreugrafia funciona como uma fonte emissora de raios $\mathrm{X}$ e um "ecran" especial que, ao receber a emissão dos raios $\mathrm{X}$, torna-se fluorescente, permitindo-se que as imagens pulmonares sejam fotografadas por uma câmara fotográfica. Estas câmaras, mesmo utilizando filmes rápidos, exigem exposições muito maiores que aquelas necessárias para a fetiura de uma telerradiografia. Uma carga de aparelho de abreugrafia é em média 10 vezes maior que uma telerradiografia. Contudo, sabese que o controle e a fiscalização desses aparelhos em uso em nosso país deixa muito a desejar. Recentemente, em São Paulo, um grupo de especialistas discutiu exaustivamente sobre a utilização da abreugrafia, como exame de massa em saúde ocupacional**. Naquela ocasião, foi dito textualmente por um físico: "Existem atualmente em uso muitos aparelhos de abreugrafia mal regulados e que emitem até 50 vezes mais radiações que um aparelho de telerradiografia"****.

Boice e col. ${ }^{5,6}$ referem que os riscos de exposição às radiações, mesmo quando em doses baixas, é proporcional à somatória do total de doses recebidas durante a vida. Esses autores correlacionam o aumento da incidência de câncer de mama com a dose total média recebida em pacientes portadores de tuberculose que necessitavam avaliações radiológicas periódicas. Estudos feitos em duas populações diferentes foram compatíveis a despeito da dose média total recebida ter sido diferente.

No presente trabalho mostramos o resultado do censo abreugráfico de uma empresa de grande porte, para o ano de 1984, discutindo o emprego de abreugrafias periódicas em saúde ocupacional.

\section{MATERIAL E METODOS}

Os dados coletados são referentes ao censo abreugráfico de 1984 , para uma população de 7.619 funcionários de uma empresa do ramo de serviços, isentos de risco respiratório de monta. Os dados estão sumarizados na Tabela 1.

TABELA 1

Abreugrafia ro censo de 1984

\begin{tabular}{lrr}
\hline Situação & N. & $\%$ \\
\hline Gestantes & 111 & 1,4 \\
Funcionários recém-admitidos & 112 & 1,4 \\
*Ausentes & 235 & 3,0 \\
Menores & 9 & 0,1 \\
Abreugrafias com validade & 1249 & 16,3 \\
Abreugrafias não realizadas & & \\
- Telerradiografias & 66 & 0,9 \\
- Não se submeteram ao exame & 529 & 6,9 \\
Abreugrafias realizadas & 5308 & 69,6 \\
\hline Total & 7619 & 100 \\
\hline
\end{tabular}

* Ausentes: Funcionários que no momento do censo encontravam-se em situação especial (licenças diversas, disponibilidade, entre outras).

As abreugrafias foram realizadas por dois serviços particulares, o primeiro deles utilizando-se de um aparelho Salgado Hermes de 200.000 amperes e filmes $70 \mathrm{~mm}$ Kodak ou Fuji. A leitura abreugráfica foi feita por especialistas em cardiologia e radiologia. O segundo serviço

* Circular enviada em 18-1-1985, pelas Secretarias de Relações do Trabalho e de Segurança e Medicina do Trabalho, do Ministério do Trabalho, aos respectivos órgãos.

** Reunião realizada na FUNDACENTRO.

*** Coelho, G.M. Comunicação pessoal — Junho/1984 
ALGRANTI, E. et al. A inadequação dos exames radiológicos periódicos indiscriminados em saúde ocupacional: resultados do censo de 1984 em uma empresa de grande porte. Rev. Saúde públ., S. Paulo, 20: 26-32, 1986.

utilizou-se de um aparelho Salgado Hermes - Generay de 100.000 amperes, e filmes de $70 \mathrm{~mm}$ Sakura ou Fuji, com laudos fornecidos por um pneumologista.

Os custos são expressos em ORTN para um idéia atual dos gastos.

\section{RESULTADOS}

De um total de 5.308 abreugrafias, 53 (1\%) foram dadas como anormais. Estes 53 funcionários submeteram-se a telerradiografia de tórax; os resultados encontram-se na Tabela 2.

TABELA 2

Resultados das telerradiografias de funcionários que tiveram anormalidades constatadas nas abreugrafias periódicas

\begin{tabular}{cccccccc}
\hline Resultados & Normal & TB ativa & $\begin{array}{c}\text { Lesões } \\
\text { residuais }\end{array}$ & $\begin{array}{c}\text { Alterações } \\
\text { c. vasculares }\end{array}$ & $\begin{array}{c}\text { Alterações } \\
\text { col. dorsal }\end{array}$ & outros & Total** \\
\hline$N^{\circ}$ & 13 & $4 *$ & 12 & 5 & 3 & 8 & 45 \\
\hline
\end{tabular}

* $3 / 4$ casos foram bacilíferos

* Dos 53 casos que apresentaram anormalidades abreugráficas, 8 telerradiografias não foram localizadas nos arquivos. Podemos afirmar que nenhuma destas 8 chapas revelaram lesōes compatíveis com tuberculose ativa ou residual devido às fichas dos funcionários.

A Tabela 3 revela os custos diretos do censo de 1984.

Depreende-se destes resultados que o rendimento do censo para a detecção de tuberculose ativa foi de $4 / 5.308$ abreugrafias, ou $0,75 / 1.000$ abreugrafias realizadas.
Os 4 casos de tuberculose ativa apresentavam quadro clínico compatível com a infecção, sendo portanto passíveis de diagnóstico por procura espontânea de serviços médicos.

TABELA 3

Custos diretos (em ORTN) do censo abreugráfico de 1984

\begin{tabular}{cccccc}
\hline Realizadas & $\begin{array}{c}\text { Custo } \\
\text { total }\end{array}$ & $\begin{array}{c}\text { Custo } \\
\text { unitário } \\
\text { médio }\end{array}$ & $\begin{array}{c}\text { Custo médio para } \\
\text { casos suspeitos (53) }\end{array}$ & $\begin{array}{c}\text { Custo médio para } \\
\text { diag. de TB ativa }\end{array}$ & $\begin{array}{c}\text { Custo médio } \\
\text { para o diag. de } \\
\text { TB ativa bacilífera }\end{array}$ \\
\hline 5.308 & $1.025,29$ & 0,19 & 19,34 & 256,32 & 341,76 \\
\hline
\end{tabular}

\section{DISCUSSÃO}

Um dos dados que chama atenção, refere-se ao número de funcionários que não se submeteram a abreugrafia, ou que apresentaram uma telerradiografia em substituição. Estes dois grupos somados chegam a $7,8 \%$ dos funcionários ativos na época do censo. As razões que levaram a não realização da abreugrafia devem ser diversas, porém acreditamos que o reconhecimento da nocividade das radiações venha sendo crescentemente familiar ao meio leigo, como mostram as repetidas reportagens na imprensa escrita. Como curiosidade, notamos que em 
ALGRANTI, E. et al. A inadequação dos exames radiológicos periódicos indiscriminados em saúde ocupacional: resultados do censo de 1984 em uma empresa de grande porte. Rev. Saúde públ., S. Paulo, 20: 26-32, 1986.

1982 a percentagem de não comparecimento aos exames era de $1,6 \%$.

Os custos do censo nos parecem extremamente altos, principalmente se levarmos em conta que os 4 casos de tuberculose ativa eram sintomáticos, com grande possibilidade de terem confirmado o diagnóstico pela procura espontânea de serviços médicos.

E difícil termos uma estimativa aproximada do chamado "custo indireto" do censo, que inclui a perda de tempo de serviço para a realização das abreugrafias. Obviamente, caso os pacientes fossem previamente selecionados pelo exame clínico, os custos indiretos seriam sensivelmente reduzidos.

O grande trunfo dos exames radiológicos em massa é a detecção da tuberculose. E claro que outras patologias torácicas têm a chance de ser diagnosticadas por tais exames, nenhuma delas, até o momento, justificando a sua utilização. Em 1960, a Comissão de Especialistas em Tuberculose da OMS ${ }^{7}$ afirmava que os exames radiológicos em massa, além de pouco específicos, incorrem em um alto custo devido ao grande número de exames necessários para o diagnóstico de um caso novo de tuberculose. Villas Boas ${ }^{18}$ e Toman ${ }^{16}$, comentando estudos de exploração radiológica em massa para tuberculose, mostram dados de taxas de detecção de até $24 \%$ dos casos novos de tuberculose bacilífera.

O uso de abreugrafias ou telerradiografias indiscriminadas em saúde ocupacional, como meio de identificação da tuberculose e outras patologias torácicas nos exames admissionais e periódicos, tem recebido um enfoque pequeno na literatura. Em 1961, Figueira e Assunção ${ }^{9}$, analisando os resultados de detecção de tuberculose por unidade abreugráfica do SESI (Serviço Social da Indústria), em Minas Gerais, mostraram taxas de 10 a $22 / 1.000$ casos suspeitos de tuberculose em candidatos a admissão à indústria. Neste levantamento deve haver diversos casos de exames duplicados em candidatos que mudavam de empresa em curto espaço de tempo. Calcados nestes dados, aqueles autores advogavam o uso da abreugrafia como exame pré-admissional necessário.

Gikovate e Nogueira 10 enfatizam, entre outros tópicos, o problema das radiações nos exames abreugráficos, concluindo que além de se tratar de exames de alto custo e de baixa rentabilidade revestem-se de um potencial tóxico, ignorado e subestimado por quem os emprega. Neste trabalho são apresentados dados dos exames periódicos em uma indústria textil de São Paulo, para os anos de 1952 e 1960 , com taxas de tuberculose bacilifera flutuando entre 0 a 6,5/1.000.

Mendes ${ }^{12}$ analisando as implicações do emprego da abreugrafia em tuberculose e higiene e medicina do trabalho, após comentar a bibliografia sobre o tema conclui que "carece totalmente de sentido permanecer em nossa legislação a obrigatoriedade deste exame, tal como a expressa no artigo 169 da CLT". Como alternativas, são propostos exames radiológicos em grupos de riscos, a baciloscopia em sintomáticas respiratórias e a triagem tuberculínica prévia em algumas eventualidades.

Jafferian e col. ${ }^{11}$ mostraram a importância de um programa de controle da tuberculose nos funcionários de um hospital público de São Paulo. E interessante notar que nestes trabalhos a exploração inicial foi feita através da prova tuberculínicia, com telerradiografia de tó$\operatorname{rax}$ nos reatores fortes. As taxas de detecção foram de $1,9,3,5$ e 1,2/1.000 para os anos de 1975,1976 e 1977 , respectivamente. 
ALGRANTI, E. et al. A inadequação dos exames radiológicos periódicos indiscriminados em saúde ocupacional: resultados do censo de 1984 em uma empresa de grande porte. Rev. Saúde públ., S. Paulo, 20: 26-32, 1986.

Nos Estados Unidos os exames radiológicos periódicos em saúde ocupacional foram revistos pela "American Occupational Medical Association" 3. A radiologia torácica rotineira foi considerada injustificável em atividades sabidamente não causadora de doenças pulmonares. Nos exames admissionais, a radiologia tóracica seria aceita desde que houvesse uma justificativa baseada na história clínica e profissional, no exame clínico e na atividade proposta. Nos exames periódicos, a solicitação seria feita baseada na ocorrência ou não de exposição ocupacional a agentes sabidamente tóxicos ${ }^{15}$. Conclusões semelhantes são publicadas por Ashenburg ${ }^{4}$.

Um estudo sobre a validade das abreugrafias periódicas foi realizado em um serviço de medicina do trabalho de uma repartição pública de Paris: Ameille e col. ${ }^{2}$ descrevem os resultados de 137.256 abreugrafias realizadas entre 1975 e 1979. A baixa proporção de casos de tuberculose (bacilífera ou não) detectados, $0,39 / 1.000$, assim como de outras patologias torácicas, levou os autores a concluir que os exames radiológicos de tórax em massa devem ser adaptados em função dos riscos ambientais e do risco individual de cada empregado.

Resultados semelhantes são apresentados por Ficheroulle e Morel ${ }^{8}$ referentes a exames abreugráficos periódicos nos anos de 1978, 1979 e 1980, nas localidades de Roubaix-Turcoing, Canadá. De um total de 100.369 abreugrafias foram descobertos 66 casos de tuberculose, $0,66 / 1.000$, e um total de $1,88 / 1.000$ de alterações abreugráficas diversas. Os autores interpretavam como importantes os resultados obtidos e que justificavam o emprego do procedimento, porém assinalam que estes exames sejam adequados ao risco profissional e a situação epidemiológica da região.
Verrastro e col. ${ }^{17}$, analisando 26.394 abreugrafias numa empresa privada na região da Grande São Paulo, descrevem um total de 371 alteraçôes, das quais 29 $(1,4 / 1.000)$ eram de tuberculose ativa bacilífera ou abacilífera. Dentre as 371 alterações incluem-se 100 casos de alterações de esqueleto ( 85 casos de cifo-escoliose), dos quais apenas um caso era significativo (plasmocitoma). Os autores concluem que a abreugrafia em massa é um exame de "risco praticamente nulo e baixo custo operacional comparados com o benefício que proporciona".

A abreugrafia representa, indubitavelmente, um meio diagnóstico de grande utilidade, tanto para cadastro quanto para exame de sintomáticos respiratórios. Todo exame "em massa" deve ter como postulados uma boa relação "custo-benefício" e "risco-benefício", assim como boa sensibilidade e especificidade. Da forma com que é empregada no Brasil, não nos parece que obedeça a estes postulados. Examinar indiscriminadamente uma população economicamente ativa, constituída predominantemente por indivíduos sãos, muitas vezes em atividades isentas de riscos respiratórios, é no mínimo de alto custo e de elevado risco, em relação a uma possível detecção de patologias assintomáticas. A abreugrafia é também considerada um exame de baixa especificidade em relação a tuberculose pulmonar, quando comparada à baciloscopia $^{18}$.

A tendência médica atual é de máxima restrição à exposição humana às radiações ionizantes. O uso de abreugrafias implica na absorção de doses de tais radiações, que são superiores a uma radiografia convencional de tórax. Os aparelhos de abreugrafia devem ser vistoriados e regulados periodicamente; quando desregulados podem emitir até 25 vezes mais radiação. Isto representa para o 
ALGRANTI, E. et al. A inadequação dos exames radiológicos periódicos indiscriminados em saúde ocupacional: resultados do censo de 1984 em uma empresa de grande porte. Rev. Saúde públ., S. Paulo, 20: 26-32, 1986.

usuário emissões absorvidas de até 2500 mrem.

Tomando o rumo das atuais discussões e propostas de alteração da legislação específica, parece-nos importante apresentar alguns pontos relevantes:

a) Somos favoráveis ao exame radiológico pré-admissional quando adequados à história clínica e profissional, assim como adequados ao exame clínico do candidato. Havendo risco ambiental respiratório no trabalho proposto o exame radiológico deve ser solicitado.

b) Excetuando-se atividades de risco pneumoconiótico, poderão ser solicitados tanto abreugrafias quanto telerradiografias, na dependência dos recursos existentes na região. Tais exames devem ser realmente custeados pelo empregador.

c) Os exames periódicos devem ser objeto de cuidadosa discussão para o es- tabelecimento de atividades que obrigatoriamente exigem acompanhamento radiológico, com vistas a transformá-las em legislação explícita. Tal legislação deve ser suficientemente aberta para vir a acomodar novos conhecimentos a respeito de doenças ocupacionais respiratórias.

d) Enquanto não forem estabelecidas novas normas regulamentadoras dos exames admissionais e periódicos, estes últimos devem ficar a critério de cada empresa, tendo em base os riscos ambientais existentes, e com uma periodicidade ditada pelo conhecimento atual de tais riscos.

\section{AGRADECIMENTOS}

Ao Dr. José Carlos Brandão de Almeida Prado, Rubens Buonavoglia e Berenice Souza pela valiosa colaboração na coleta de dados.

ALGRANTI, E. et al, [The inadequacy of indiscriminate periodical X-ray exams in occupational health. The results of the 1984 census in a large firm]. Rev. Saúde públ., S. Paulo, $20: 26-32,1986$

ABSTRACT: This paper reports on the results of the 1984 Minimal Mass Radiography (MMR) screening in a large company with no respiratory harzards are reported. Among 5,308 MMR there were 53(1\%) abnormal findings with 4 cases of active pulmonary tuberculosis. The yield from screening was $0.75 / 1,000$ MMR. The mean cost of diagnosing each active case of tuberculosis was around US\$1,850. An increase in refusal rates to attend the screening, from $1.6 \%$ in 1982 to $7.8 \%$ in 1984 , probably because of the increasing lay knowledge of radiation harzards, was also noted. The results are discussed with a short revision of ionizing radiations and the usefulness of X-ray screenings in occupational health.

UNITERMOS: Mass chest X-ray. Radiation, ionizing. Occupational health. 
ALGRANTI, E. et al. A inadequação dos exames radiológicos periódicos indiscriminados em saúde ocupacional: resultados do censo de 1984 em uma empresa de grande porte. Rev. Saúde públ., S. Paulo, 20: 26-32, 1986.

\section{REFERENCIAS BIBLIOGRÁFICAS}

1. ABREU, M. Carteira de saúde universal. Rev. bras. Tuberc., 30:115-6, 1962.

2. AMEILLE, J.; CARIOU, R.; LECLERC, P.; CONSTANS, P.; DUFAT, R.; PROTEAU, J. \& ROCHE-MAURE, J. L'examen radiologique annuel du thorax est-il encore justifié en médecine du travail? Nouv. Press. med. 11:2539-42, 1982.

3. AMERICAN OCCUPATIONAL MEDICAL ASSOCIATION. Committe Report. Guidelines for use of routine X-ray examinations in occupational medicine. J. occup. Med., 21:500-2, 1979.

4. ASHENBURG, N.J. Routine chest X-Ray examination in occupational medicine. J. occup. Med., 24:18-20, 1982.

5. BOICE, J.D.; ROSENSTEIN, M. \& DALE TROUT, E. Estimation of breast doses and breast cancer risk associated with repeated fluoroscopic chest examinations of women with tuberculosis. Radiat. Res., 73:373-90, 1978.

6. BOICE, J.D.; MONSON, R.R. \& ROSENSTEIN, M. Cancer mortality in womens after repeated fluoroscopic examinations of the chest. J. Nat. Cancer Inst., 66:863-7, 1981.

7. EVALUACION de las recomendaciones formuladas por los grupos de expertos de la OMS en tuberculosis. Cron. OMS, 24:487-94, 1974.

8. FICHROULLE, X. \& MOREL, C. Despistages radiologiques pulmonaires en medicine du travail. Larc med., 3:203-5, 1983.

9. FIGUEIRA, A.W. \& ASSUNÇÃO, N. Admissão à indústria. Rev. bras. Tuberc., 29:313-29, 1961.
10. GIKOVATE, F. \& NOGUEIRA, D.P. Abreugrafia sistemática em massa: inviabilidade econômica e eventuais perigos da exposição a radiações. Rev. Saúde públ., S. Paulo, 10:103-10, 1976.

11. JAFFERIAN, P.A.; MORRONE, L.C. \& SANTOS, M.A.S. Freqüência da tuberculose entre funcionários de uma instituição de assistência médica e os resultados parciais de um programa de controle. Rev. bras. Saúde ocup., 5:30-3, 1977.

12. MENDES, R. Reavaliação da abreugrafia na luta contra a tuberculose: implicações do seu emprego em higiene e medicina do trabalho. Brasília, Ministério da Saúde. Secretaria Nacional de Ações Básicas de Saúde. Divisão de Ecologia Humana e Saúde Ambiental, 1977.

13. MINISTÉRIO DO TRABALHO. Norma Regulamentadora n..$^{\circ}$ 15. Brasília, 1978.

14. MINISTÉRIO DO TRABALHO. Norma Regulamentadora $n .^{\circ}$ 7. Brasília, 1983.

15. PODA, G.A. Chest X-Ray examination in occupational medicine. J. occup. Med., 25:773, 1983.

16. TOMAN, K. Exploración radiográfica en massa y lucha antituberculosa. Cron. OMS, 30:53-60, 1976.

17. VERRASTRO, C.; BETTI, R. \& CAMPI, R. Exame abreugráfico de rotina em empresa de grande porte. Rev. bras. Saúde ocup., 11:19-24, 1983.

8. VILLAS BOAS, A. Descobrimento de casos de tuberculose. Rev. Div. nac. Tuberc. 18:16-32, 1974.

Recebido para publicação em 08/08/1985 Aprovado para publicação em 21/10/1985. 